\title{
La necesidad ontológica del pensamiento salvaje. Desarrollo y aprendizaje en la teoria de la percepción de Maurice Merleau-Ponty. Aportes y discusiones en torno a una fenomenologia del esquema corporal en la primera infancia
}

\section{( Jesica Estefanía Buffone}

Universidad de Buenos Aires/ Université Jean Moulin-Lyon 3/ Universidad Nacional de San Martín/Consejo

Nacional de Investigaciones Científicas y Técnicas, Argentina

Directores: Dra. Graciela Ralón de Walton (Universidad Nacional de San Martín) - Dr. Etienne Bimbenet (Lyon 3)

Co-directora: Dra. Mariana Larison

Jurado: Dr. Maximiliano Cladakis, Dr. Esteban García, Dr. Emmanuel de Saint Aubert, Dr. Yves Thierry.

Fecha de defensa: 22 de noviembre de 2018

El análisis fenomenológico de la experiencia que Maurice Merleau-Ponty comienza a esbozar en La estructura del comportamiento (1942) y al que da forma en la Fenomenología de la percepción (1945) nos pone frente a la necesidad de volver al mundo de la vida reivindicando el contacto prístino que tenemos con las cosas, con los otros y con nosotros mismos. En la necesidad de regresar a ese mundo desde una mirada ingenua y enquistada en nuestra historia es que podemos encontrar la necesidad misma de un análisis fenomenológico de la infancia, la cual es en la obra de Merleau-Ponty no solo un período en el desarrollo psicogenético de un sujeto, sino una figura cuasi literaria, poética, que nos sitúa en los inicios de la dinámica misma de ser arrojados al mundo. La niñez, por lo tanto, como imagen representativa de la experiencia salvaje oculta por el halo del cientificismo y como estadio concreto en el desarrollo psicomotor de un sujeto, aparecerá en la obra del filósofo francés para describir una nueva concepción de la subjetividad humana, atravesada por el mundo y en constante diálogo con él.

El objetivo general de la tesis es analizar los procesos que intervienen en la construcción del esquema corporal durante la primera infancia, para así reconstruir la génesis del aparato perceptivo desde una perspectiva fenomenológica. ¿Existe alguna diferencia entre la teoría formulada en la Fenomenología de la percepción y la concepción que sobre este proceso se sostiene en los cursos dedicados a la infancia? ¿Es el esquema corporal un constructo que se organiza y redefine a partir de dinámicas similares al proceso histórico mismo? La propiocepción, la imaginación, el traspaso postural y la construcción de las habitualidades perceptivas son algunos de los procesos que serán cotejados críticamente en el seno de su teoría, para dilucidar la apropiación y la utilización que de las mismas realiza el filósofo francés y para unirlas, al mismo tiempo, con algunos debates actuales de la psicología del desarrollo. Asimismo, los procesos de constitución y organización perceptiva serán puestos en diálogo con la dinámica que Merleau-Ponty describe como subyacente a la temporalidad histórica, dilucidando los quiebres y las similitudes entre la definición del cuerpo propio y el relanzamiento de la historia misma. De esta manera, uno de los objetivos específicos del presente trabajo es explorar no solamente las posibilidades que la concepción de la infancia de Maurice Merleau-Ponty ofrece para analizar algunos problemas vigentes de la psicología infantil, sino que también se indagarán los derroteros políticos que su teoría corporal de la percepción parece habilitar desde su definición misma de cuerpo.

\section{Contenido}

En el capítulo 1 de la primera parte de la tesis, se llevó adelante el análisis de la apropiación que realiza Merleau-Ponty de algunos conceptos centrales de la Gestalt propuestos por Koffka y Köhler, a partir de los cuales redefinió un nuevo rumbo en el estudio de la infancia. En el capítulo 2, se realizó un análisis de algunas de las obras de Maurice Merleau-Ponty en las que el fenomenólogo brinda una descripción de la infancia o en donde la imagen del niño vendrá a dar cuenta de algún aspecto relevante dentro de su teoría. A partir de este análisis, se ha evidenciado cómo la infancia no es solo un objeto de interés metodológico en sus estudios de psicología, sino que también representa ese ir-hacia primigenio de la experiencia, la pura potencia que aún no se ha arrojado en el mundo. En el capítulo 3, se ha analizado (tomando como referencia algunos cursos de Psychologie et 
pédagogie de l'enfant. Cours de Sorbonne) la explicación que realiza Merleau-Ponty del desarrollo infantil a partir de la dinámica histórica propia del marxismo, en la cual la obra de Henri Wallon se presenta como el horizonte de la discusión. La redefinición del concepto de campo fenomenal en términos de situación atravesada por las condiciones materiales de existencia de un sujeto será central para comprender la organización tónico postural.

En la segunda parte de la tesis se ha explorado la construcción y organización corporal en la primera infancia desde la redefinición del cuerpo como esquema, explorando algunos de los conceptos centrales que dentro de la teoría merleau-pontyana intervienen en este proceso. En el capítulo 1, se abordan los antecedentes de los que se nutre Merleau-Ponty para construir su caracterización de la corporalidad como esquema, esto es, como cuerpo vivido y construido intersubjetivamente. En el capítulo 2, se llevó a cabo un análisis de la apropiación que el filósofo francés realiza del concepto de esquema corporal, para así dilucidar las implicancias que el mismo posee en la descripción que elabora de la construcción del cuerpo propio en la primera infancia, haciendo hincapié en el rol que la impregnación postural, la propiocepción y la imaginación poseen en la construcción del cuerpo en el comienzo de la vida del niño. Las sensaciones propioceptivas experimentadas durante los primeros meses de vida serán el fundamento del accouplement (el emparejamiento corporal), el cual se postula como la base del entrelazo con los otros que experimentaremos a lo largo de nuestra vida. En el capítulo 3, se ha explorado la génesis de la función Ilamada arco intencional, asumiéndola como una función de paulatino desarrollo y analizando los procesos intermedios (como la constitución del yo y la aparición del mundo de los objetos) que colaboran en su definición. Asimismo, se ha evidenciado que el concepto de arco intencional, aunque escasamente desarrollado por Merleau-Ponty, conlleva consecuencias que impregnan y sostienen gran parte de su teoría.

En la tercera parte, se analizó la vida afectiva de un sujeto como matriz a partir de la cual se cristalizan determinadas formas de sociabilidad. La ligazón entre afectividad y funciones cognitivas se instala como el motor del desarrollo, determinando nuestra manera de asir y resignificar el mundo circundante. El capítulo 1 es una indagación sobre el rol que los sentimientos poseen dentro de la teoría merleaupontyana. En primer lugar, se abordó el análisis que Merleau-Ponty realiza en Fenomenología de la percepción (1945) en torno a la existencia de sentimientos falsos e ilusorios, y la forma en la que logra rebatir la posición cartesiana que los descarta. El sentimiento será crucial en el desarrollo intelectual y en la construcción que el niño (y más tarde el adulto) harán del mundo y de las relaciones circundantes. De esta manera, el sentimiento, en tanto afección ligada a lo irracional y transitorio, se ve vinculada no solo con el desarrollo intelectual, sino que determinará la ligazón que mi cuerpo tendrá con el mundo y con las cosas. En el capítulo 2, se analizó el papel que desempeña el complejo de Edipo en la fenomenología de Merleau-Ponty, para comprender a partir de allí otros eventos considerados como instituyentes en la vida personal de un individuo, tales como el enamoramiento o el nacimiento. En el marco de un análisis comparativo entre, por un lado, La institución en la historia personal y pública, y, por otro, L'enfant vu par l'adulte y Les relations avec autrui chez l'enfant, el Edipo se presenta como una dinámica instituyente en la medida en que abre al niño por vez primera a una nueva sociabilidad, a partir de la cual sus hábitos perceptivos comenzarán a reelaborarse. Su historia personal y la herencia filogenética se entrelazan así para resignificarse mutuamente.

En la cuarta parte, se abordan algunos tópicos centrales de la psicología del desarrollo (tales como la aparición de los objetos en el proceso comunicacional y el desarrollo de la temporalidad infantil) a partir de la filosofía de Merleau-Ponty. En el capítulo 1, se explora desde una perspectiva fenomenológicocorporal el tránsito entre la intersubjetividad primaria y la intersubjetividad secundaria (esto es, cómo se da el pasaje entre la interacción puramente diádica y aquella que incluye el mundo de los objetos). Para ello, se llevó a cabo un análisis de la construcción de las habitualidades motrices, poniendo en el centro de la discusión la ligazón del cuerpo con las otras subjetividades y con los objetos culturales como polos que definen nuestra aproximación al mundo circundante. En el capítulo 2, se analizó el desarrollo de la noción de temporalidad en la primera infancia desde una perspectiva fenomenológica, postulando al cuerpo y a la manipulación de los objetos en el mundo como factores cruciales en este proceso. La experiencia estética infantil es presentada por Merleau-Ponty como eminentemente fenomenológica, como la representación del tiempo vivido que aún no ha sido asimilada por la culturalización y las formas estandarizadas de vivir el tiempo y el espacio.

\section{Palabras finales}

La banalización de la infancia y el borramiento de su enquistamiento en la historia ha sido el precio 
que la minoría de edad tuvo que pagar para formar parte de los temas de estudio de la ciencia, por lo que el llamamiento de Merleau-Ponty de resguardar a la niñez del avance irrefrenable del pensamiento formal (crítica que es personificada en la figura de Piaget), no es tanto un llamamiento metodológico como un imperativo ético de la investigación científica y filosófica. "Volver a las cosas mismas" no será para el fenomenólogo francés meramente el legado de una tradición filosófica que da cuenta de una falla epistemológica en la producción científica. “Volver a las cosas mismas" reviste un valor ético que va más allá de lo metodológico y que debe mantenerse como horizonte de investigación para no incurrir en el olvido de las particularidades históricas y culturales de los sujetos que se construyen como objetos de estudio. Por esa razón, esta tesis ha intentado resituar la infancia, a las infancias, en el contexto que les es inherente, pudiendo comprenderlas desde allí, haciendo de las vicisitudes históricas y de los lazos que las traspasan, una parte vital para su entendimiento. En ese sentido, cuando Merleau-Ponty afirmaba que era preciso salvar a los niños de Piaget, nos estaba previniendo del olvido y estaba anunciando la inminente llegada y consolidación de una mirada cientificista y naturalista que haría del divorcio de la experiencia, del desmedro de los sentidos, la bandera irrefrenable con la que se consolidaría en el ámbito académico. En medio de esa coyuntura, el legado merleau-pontyano adquiere una dimensión que se desborda de los objetivos explícitos en su obra: su teoría corporal de la percepción (según la cual nuestras conductas se organizan y consolidan en medio de una relación dialéctica con el mundo) da cuenta de una implicación efectiva entre nuestro cuerpo y las circunstancias socio-históricas que nos envuelven, a partir de su descripción de la adquisición de habitualidades perceptivas como mediadas por los objetos y por los otros. Nuestra manera de "ir hacia las cosas mismas" es un reflejo de nuestra imbricación con el entorno, una reelaboración de las condiciones materiales que nos envuelven. Una teoría corporal de la percepción como la descripta por Maurice MerleauPonty deja al descubierto las connotaciones políticas que posee hacer de nuestro cuerpo el vehículo de una temporalidad determinada.

Volver a las cosas mismas, entonces, se nos presenta como una necesidad ética, política y metodológica para que los sujetos de la experiencia no sean nuevamente sepultados, para que el manto de la universalidad artificial no haga del mundo de la vida un ámbito borroso, y del mundo de la ciencia, un discurso estéril. 